\title{
PROPAGACIÓN VEGETATIVA DE PLÁTANO Y BANANO CON LA APLICACIÓN DE BENZILAMINOPURINA (6-BAP) Y ACIDO INDOLACETICO (AIA)
}

\author{
Hayron Fabricio Canchignia Martínez ${ }^{1}$, Marjorie Dolores Espinoza Roca ${ }^{2}$, Gonzalo Rafael Benavides Velasquez², \\ Silvia Gicela Saucedo Aguiar ${ }^{1}$, Mercedes Susana Carranza Patiño ${ }^{3}$ y Orly Fernando Cevallos Falquez ${ }^{4}$ \\ ${ }^{1}$ Unidad de Investigación Científica y Tecnológica, Facultad de Ciencias Agrarias, Universidad Técnica Estatal de Quevedo. \\ Av. Walter Andrade. Km 1 11/2 vía a Santo Domingo, C.P. 73. Quevedo, Los Rios, Ecuador. 099300382. hfcanma@yahoo.es. \\ ${ }^{2}$ Facultad de Ciencias Agrarias, Universidad Técnica Estatal de Quevedo. Quevedo, Los Ríos, Ecuador \\ ${ }^{3}$ Unidad de Investigación Científica y Tecnológica, Facultad de Ciencias Ambientales, Universidad Técnica Estatal de \\ Quevedo. Quevedo, Los Ríos, Ecuador \\ ${ }^{4}$ Unidad de Investigación Científica y Tecnológica, Facultad de Ciencias Pecuarias, Universidad Técnica Estatal de \\ Quevedo. Quevedo, Los Ríos, Ecuador.
}

\section{RESUMEN}

En la propagación vegetativa de dos variedades de Banano (Valery y Orito) y una de Plátano (Barraganete), se sometió a diferentes tratamientos hormonales, para evaluar el número de brotes, la concentración $30 \mathrm{mg} \mathrm{L}^{-1}$ de BAP alcanzó el mayor promedio, con 2.36 brotes para todas las variedades. En longitud y diámetro de brotes, la concentración que logró el mayor promedio fue $\mathrm{C}_{0}$, no encontrándose diferencias entre las concentraciones de 6-BAP y AIA. La variedad que presentó la mayor longitud y diámetro de brotes fue el banano Orito con 55.65 y $2.97 \mathrm{~cm}$, respectivamente. El mayor porcentaje de vigor alto de brote lo demostró el tratamiento con $40 \mathrm{mg} \mathrm{L}^{-1} \mathrm{BAP}+12 \mathrm{mg} \mathrm{L}^{-1}$ AIA con 24.17\%; el mayor porcentaje de vigor medio lo alcanzó el tratamiento con $30 \mathrm{mg} \mathrm{L}^{-1}$ BAP y sin hormona con $72.22 \%$, la supervivencia de cepas, en las variedades Barraganete y Orito presentaron el 100\% de cepas vivas, superando al banano Valery quien obtuvo un promedio de $91.67 \%$.

Palabras clave: Benzilaminopurina (6-BAP), ácido indolacetico (AIA).

\begin{abstract}
In the vegetative propagation of two varieties of Banana (Valery and Orito) and one of Palntein (Barraganete), it was put under different hormonal treatments, to evaluate the number of buds, concentration $30 \mathrm{mg} \mathrm{L}^{-1}$ of BAP reached the greater average, with 2.36 appear for all the varieties. In length and diameter of buds, the concentration that obtained the greater average was $\mathrm{C}_{0}$, not being differences between the concentrations of 6-BAP and AIA. The variety that showed the greater length and diameter of buds was the Orito banana tree with 55.65 and $2.97 \mathrm{~cm}$, respectively. The greater percentage of high vigor of bud was $40 \mathrm{mg} \mathrm{L}^{-1} \mathrm{BAP}+12 \mathrm{mg} \mathrm{L}^{-1}$ AIA with $24.17 \%$; the greater percentage of average vigor reached $30 \mathrm{mg} \mathrm{L}^{-1} \mathrm{BAP}$ and without hormone with $72.22 \%$. The survival of stocks the varieties Barraganete and Orito presented/displayed the $100 \%$ of alive stocks, surpassing to the banana tree Valery who obtained an average of $91.67 \%$.
\end{abstract}

Key words: Benzilaminopurine (6-BAP), indolacetic acid (AIA).

\section{INTRODUCCIÓN}

La carencia de material de alta calidad es uno de los factores que limitan el buen desarrollo de las plantaciones de plátano y banano. La propagación vegetativa consiste en la estimulación y proliferación de brotes mediante la aplicación exógena de reguladores de crecimiento. La propagación clonal o vegetativa para Vásquez et al. (2000), es un método utilizado para multiplicar partes vegetativas, utilizándose tejidos vegetales que conserven las características hereditarias de planta donadora y así generar nuevos individuos. Taiariol (2000), la propagación vegetativa o asexual se utiliza para producir una planta que posea el mismo genotipo que la planta madre (planta donadora) y esto

Recibido: Mayo, 2007. Aceptado: Noviembre: 2007

Publicado como ARTÍCULO en Ciencia y Tecnología 1: 11-15. 2008. es posible porque todas las células de una planta poseen la información necesaria o suficiente para reproducir la planta entera. Maluenda y Reyes (2003), manifiestan que las auxinas influyen en el crecimiento de órganos vegetales estimulando la elongación o alargamiento de ciertas células e inhibiendo el crecimiento de otras, en función de la cantidad de auxina en el tejido vegetal y su distribución. Por otro lado Bidwell (1993), en la mayoría de los procesos en que están implicadas las citoquininas ésta a su vez participa junto con otras hormonas, especialmente auxinas. La investigación tuvo como fin proporcionar una alternativa que facilite la disponibilidad de un material de siembra en óptimas condiciones (características deseables, excelente calidad genética y aspecto fitosanitario, entre 
otros) que simplifique la instalación de un sistema de producción, obteniendo plantas uniformes y permita realizar labores de cosecha de la manera más eficiente.

\section{MATERIALES Y MÉTODOS}

\section{Material vegetal}

Se colectaron cepas de plátano variedad Barraganete (Musa paradisiaca $A A B$ ), banano variedad Valery (Musa sapientum $A A A$ ), banano variedad Orito (Musa acuminata AA).

\section{Reactivos}

Benzilaminopurina (6-BAP); Acido Indolacetico (AIA), procediendo a suministrar una cantidad de 8 cc por cada cepa, de la concentración inicial de cada tratamiento.

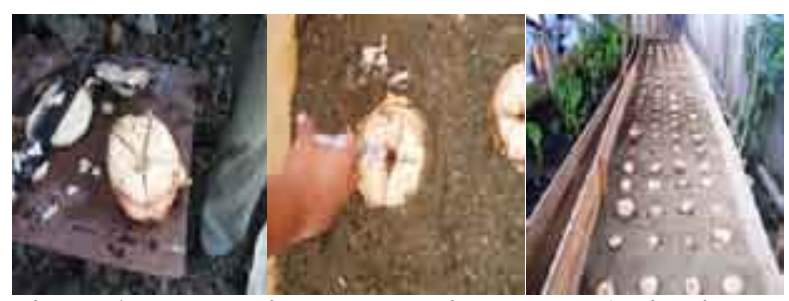

Figura 1. Preparación del material vegetal, Aplicación de las hormonas (6-BAP, AIA) y siembra

\section{Diseño Experimental, Factores Estudiados y Tratamientos}

Se utilizó un diseño de "bloques completamente al azar" con un arreglo factorial $3 \times 9$, con tres repeticiones. Cada unidad experimental estuvo constituida por cuatro cepas.

Factores de estudio. Factor A: Variedades de Banano y Plátano: $\mathrm{V}_{1}=$ Plátano Barraganete; $\mathrm{V}_{2}=$ Banano Valery; $\mathrm{V}_{3}=$ Banano Orito. Factor B: Hormonas: Se emplearon nueve concentraciones hormonales: (BAP) y (AIA) según se detalla a continuación. $\mathrm{C}_{0}=$ Sin Hormona; $\mathrm{C}_{1}$ $=30 \mathrm{mg} \mathrm{L}^{-1} \mathrm{BAP} ; \mathrm{C}_{2}=40 \mathrm{mg} \mathrm{L}^{-1} \mathrm{BAP} ; \mathrm{C}_{3}=50 \mathrm{mg} \mathrm{L}^{-1}$ $\mathrm{BAP} ; \mathrm{C}_{4}=60 \mathrm{mg} \mathrm{L}^{-1} \mathrm{BAP} ; \mathrm{C}_{5}=30 \mathrm{mg} \mathrm{L}^{-1} \mathrm{BAP}+10$ $\mathrm{mg} \mathrm{L}^{-1} \mathrm{AIA} ; \mathrm{C}_{6}=40 \mathrm{mg} \mathrm{L}^{-1} \mathrm{BAP}+12 \mathrm{mg} \mathrm{L}{ }^{-1} \mathrm{AIA} ; \mathrm{C}_{7}$ $=50 \mathrm{mg} \mathrm{L}^{-1} \mathrm{BAP}+14 \mathrm{mg} \mathrm{L}^{-1} \mathrm{AIA} ; \mathrm{C}_{8}=60 \mathrm{mg} \mathrm{L}^{-1} \mathrm{BAP}$ $+16 \mathrm{mg} \mathrm{L}^{-1}$ AIA.

\section{Datos registrados y método de evaluación}

A los 55 días después de la siembra se registraron las siguientes variables: Número de brotes por cepa, Longitud de brotes $(\mathrm{cm})$, Diámetro de brotes $(\mathrm{cm})$, Vigor de brotes.

\section{Manejo del experimento}

Construcción de vivero: $\mathrm{El}$ vivero tuvo unas dimensiones de $8 \mathrm{~m}$ de longitud por $5 \mathrm{~m}$ de ancho, constituido por tres camas de $1.40 \times 8 \mathrm{~m}$ de longitud $\mathrm{y}$ ancho, respectivamente. El vivero fue cubierto con Polietileno de alta densidad (plástico) y Zarán (25\%) que permitió reducir la intensidad luminosa y controlar la temperatura.

Preparación de concentraciones hormonales: Se procedió a pesar las distintas concentraciones de BAP y AIA; fue diluida en alcohol al (90\%); al momento de utilizar las concentraciones hormonales, cada una fueron enrasadas hasta $1000 \mathrm{cc}$ con agua destilada.

Selección del material vegetativo: Se seleccionó plantaciones de plátano y banano localizadas en los cantones Quevedo y La Maná y en el recinto El Vergel. Se empleo 108 cepas para cada variedad, seleccionadas de acuerdo a características fenotípicas como: altura de la planta donadora 1.00 a $1.50 \mathrm{~m}$, peso de cepa $2 \mathrm{~kg}$ aproximadamente y óptimo aspecto fitosanitario.

Sustratos empleados: Los sustratos en la siembra de las cepas fueron carboncillo de arroz y arena, en una proporción de $1: 1$ ( $50 \%$ cada uno).

Preparación y siembra del material vegetativo: Se procedió a lavar, eliminar raíces y pseudotallo viejo. Se corto transversalmente el pseudotallo de cada yema a $2 \mathrm{~cm}$ del cuello del rizoma; luego se eliminó la yema central o ápice meristemático (romper la dominancia apical) a una profundidad de $4 \mathrm{~cm}$, dejando una cavidad de $2 \mathrm{~cm}$ de diámetro aproximadamente; a continuación se realizó un corte en forma de cruz al segmento del pseudotallo profundizando hasta el cuello del rizoma, se realizó la aplicación de las concentraciones hormonales, de 8 cc en la cavidad dejada por la extracción de la yema central.

\section{RESULTADOS}

\section{Número de brotes}

No se encontró diferencias estadísticas entre concentraciones para cada una de las variedades, sin embargo, en la variedad banano Valery con el empleo de $30 \mathrm{mg} \mathrm{L}^{-1}$ BAP y $30 \mathrm{mg} \mathrm{L}^{-1}$ BAP $+10 \mathrm{mg} \mathrm{L}^{-1}$ AIA se obtuvieron los mayores promedios de brotes por cepa de 2.3 y 2.5 y el menor número de brotes en la concentración $\mathrm{C}_{7}$ con 1.2. En el Barraganete los mejores resultados se obtuvieron en $30 \mathrm{mg} \mathrm{L}^{-1} \mathrm{BAP}$ y $60 \mathrm{mg} \mathrm{L}^{-1} \mathrm{BAP}+16 \mathrm{mg}$ $\mathrm{L}^{-1}$ AIA con un promedio de brotes por cepa de $2.7 \mathrm{y}$ 2.3 y el menor número de brotes para la concentración $\mathrm{C}_{2}$ de 1.8. El Orito bajo la concentración de $40 \mathrm{mg} \mathrm{L}^{-1}$ BAP $+12 \mathrm{mg} \mathrm{L}^{-1}$ AIA presentó la mayor cantidad de brotes con un promedio de 2.5 y una menor influencia 
de brotes en la concentración $\mathrm{C}_{4}$ (Cuadro 1). Se reportó diferencias estadísticas, al agrupar todas las Musas, frente a cada una de las concentraciones hormonales, presentando el mayor número de brotes de 2.36 en la concentración de $30 \mathrm{mg} \mathrm{L}^{-1} \mathrm{BAP}$ (Cuadro 2 ).

\section{Longitud de brotes}

Se reportó diferencias estadísticas para las distintas concentraciones hormonales, frente a cada una de las variedades de Musas sp. En Valery el testigo y la concentración $50 \mathrm{mg} \mathrm{L}^{-1} \mathrm{BAP}+14 \mathrm{mg} \mathrm{L}^{-1}$ AIA, alcanzaron los mayores promedios de longitud por brote con valores de 41.4 y $44.1 \mathrm{~cm}$. El Barraganete mostró un mayor promedio de $57.4 \mathrm{~cm}$ de longitud en la concentración $50 \mathrm{mg} \mathrm{L}^{-1}$ BAP. En Orito su mejor resultado se presentó en $50 \mathrm{mg} \mathrm{L}^{-1} \mathrm{BAP}+14 \mathrm{mg} \mathrm{L}^{-1}$ AIA y el testigo con promedios de 59.1 y $62.4 \mathrm{~cm}$ de longitud de brotes (Cuadro 1). Se reportó diferencias estadísticas al reunir las tres Musáceas frente a cada una de las concentraciones hormonales de (6-BAP y AIA), obteniendo el mayor promedio de longitud por brotes en el testigo de $51.25 \mathrm{~cm}$, siendo el coeficiente de variación de $18.21 \%$ (Cuadro 2).

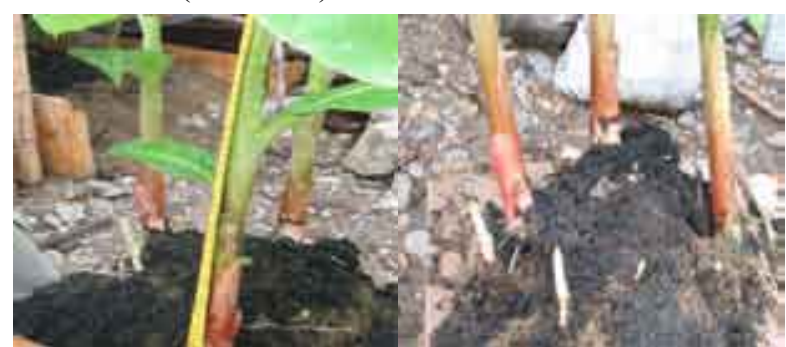

Figura 2. Longitud de brotes por concentraciones de 6-BAP y AIA, en la propagación vegetativa de plátano y banano

\section{Diámetro de brotes}

No se encontró diferencias estadísticas entre las distintas concentraciones, frente a cada una de las variedades de Musas sp. En banano Valery, empleando el testigo y la concentración $50 \mathrm{mg} \mathrm{L}^{-1} \mathrm{BAP}+14 \mathrm{mg}$ $\mathrm{L}^{-1}$ AIA se alcanzaron promedios de $3.2 \mathrm{~cm}$ de diámetro por brotes. En el plátano Barraganete se obtuvieron los mejores resultados para el testigo y la concentración $50 \mathrm{mg} \mathrm{L}^{-1}$ BAP con valores de $3.3 \mathrm{~cm}$ de diámetro por brote. El banano Orito alcanzó un mayor diámetro por brote para las concentraciones $30 \mathrm{mg} \mathrm{L}^{-1} \mathrm{BAP}+10 \mathrm{mg}$ $\mathrm{L}^{-1}$ AIA y $60 \mathrm{mg} \mathrm{L}^{-1}$ BAP con valores de 3.2 y $3.4 \mathrm{~cm}$ de diámetro (Cuadro 1). Se reportó diferencias estadísticas, al reunir las tres Musáceas frente a cada una de las concentraciones hormonales de (6-BAP y AIA) en donde el mayor promedio de diámetro se alcanzó para el testigo con $3.09 \mathrm{~cm}$, siendo el Coeficiente de Variación 16.11\% (Cuadro 2).

\section{Supervivencia de cepas}

No se encontró diferencias estadísticas entre concentraciones para cada una de las variedades, demostrando el banano Orito y plátano Barraganete el $100 \%$ de supervivencia de cepas, sin diferir estadísticamente del banano Valery que alcanzó el 91.67\% (Cuadro 1). Agrupando las tres variedades de Musas, frente a las distintas concentraciones hormonales no se reportó diferencias estadísticas en donde el $\mathrm{C}_{0}, \mathrm{C}_{2}$ $\mathrm{y} \mathrm{C}_{7}$ obtuvieron el $100 \%$ de promedio porcentual de sobrevivencia de cepas, las restantes concentraciones alcanzaron porcentajes entre $94.44 \%$ y $97.22 \%$, respectivamente (Cuadro 2).

Cuadro 1. Promedios de número de brotes; longitud de brotes $(\mathrm{cm})$; diámetro de brotes $(\mathrm{cm})$; supervivencia $(\%)$ de cepas registrados en la propagación vegetativa de Plátano y Banano con la aplicación de BAP y AIA

\begin{tabular}{|c|c|c|c|c|c|c|c|c|c|c|c|c|}
\hline & \multicolumn{3}{|c|}{ Número de brotes } & \multicolumn{3}{|c|}{ Longitud de brotes } & \multicolumn{3}{|c|}{ Diámetro de brotes } & \multicolumn{3}{|c|}{ Supervivencia } \\
\hline & Val..$^{\prime \prime}$ & Barr. ${ }^{2 /}$ & Orito & Val..$^{1 /}$ & Barr. ${ }^{2} /$ & Orito & Val..$^{\prime /}$ & Barr. ${ }^{2} /$ & Orito & Val..$^{1 /}$ & Barr. ${ }^{2}$ & Orito \\
\hline $\mathrm{C}_{0}$ & 18 & 1. & 1 & $\mathrm{~cd}$ & d & $62.36 \mathrm{a}$ & 3.0 & $\mathrm{a}$ & 3,2 & $00 \mathrm{a}$ & $\mathrm{a}$ & $100 \mathrm{a}$ \\
\hline $\mathrm{C}_{1}$ & $2.3 \mathrm{a}$ & $2.7 \mathrm{a}$ & $2.1 \mathrm{a}$ & $34.7 \mathrm{bcd}$ & $42.7 \mathrm{abcd}$ & $59.07 \mathrm{ab}$ & $2.6 \mathrm{a}$ & $2.6 \mathrm{a}$ & $2.8 \mathrm{a}$ & $83.3 \mathrm{a}$ & $100 \mathrm{a}$ & $100 \mathrm{a}$ \\
\hline $\mathrm{C}_{2}$ & $2.1 \mathrm{a}$ & $1.8 \mathrm{a}$ & $2.1 \mathrm{a}$ & $35.5 \mathrm{bcd}$ & $44.6 \mathrm{abcd}$ & $51.86 \mathrm{abcd}$ & $2.7 \mathrm{a}$ & $2.9 \mathrm{a}$ & $2.6 \mathrm{a}$ & $100 \mathrm{a}$ & $100 \mathrm{a}$ & $100 \mathrm{a}$ \\
\hline $\mathrm{C}_{3}$ & $1.8 \mathrm{a}$ & $1.6 \mathrm{a}$ & $2.2 \mathrm{a}$ & $36.9 \mathrm{abcd}$ & $57.4 \mathrm{abc}$ & $50.27 \mathrm{abcd}$ & $2.9 \mathrm{a}$ & $3.3 \mathrm{a}$ & $2.9 \mathrm{a}$ & $91.7 \mathrm{a}$ & $100 \mathrm{a}$ & $100 \mathrm{a}$ \\
\hline $\mathrm{C}_{4}$ & $2.2 \mathrm{a}$ & $2.1 \mathrm{a}$ & $1.8 \mathrm{a}$ & $30.7 \mathrm{~d}$ & 45.9 abcd & $54.73 \mathrm{abcd}$ & $2.4 \mathrm{a}$ & $2.7 \mathrm{a}$ & $3.4 \mathrm{a}$ & $83.3 \mathrm{a}$ & $100 \mathrm{a}$ & $100 \mathrm{a}$ \\
\hline $\mathrm{C}_{5}$ & $2.5 \mathrm{a}$ & $2.1 \mathrm{a}$ & $2.0 \mathrm{a}$ & $37.1 \mathrm{abcd}$ & $47.7 \mathrm{abcd}$ & $57.70 \mathrm{abc}$ & $2.8 \mathrm{a}$ & $2.8 \mathrm{a}$ & $3.2 \mathrm{a}$ & $91.7 \mathrm{a}$ & $100 \mathrm{a}$ & $100 \mathrm{a}$ \\
\hline $\mathrm{C}_{6}$ & $1.5 \mathrm{a}$ & $2.1 \mathrm{a}$ & $2.5 \mathrm{a}$ & $32.9 \mathrm{~cd}$ & $40.9 \mathrm{abcd}$ & $49.63 \mathrm{abcd}$ & $2.2 \mathrm{a}$ & $2.4 \mathrm{a}$ & $2.6 \mathrm{a}$ & $83.3 \mathrm{a}$ & $100 \mathrm{a}$ & $100 \mathrm{a}$ \\
\hline $\mathrm{C}_{7}$ & $1.2 \mathrm{a}$ & $2.1 \mathrm{a}$ & $2.2 \mathrm{a}$ & $44.1 \mathrm{abcd}$ & $41.4 \mathrm{abcd}$ & $59.10 \mathrm{ab}$ & $3.2 \mathrm{a}$ & $2.5 \mathrm{a}$ & $2.9 \mathrm{a}$ & $100 \mathrm{a}$ & $100 \mathrm{a}$ & $100 \mathrm{a}$ \\
\hline $\mathrm{C}_{8}$ & $1,4 \mathrm{a}$ & $2.3 \mathrm{a}$ & $2.2 \mathrm{a}$ & $32.7 \mathrm{~cd}$ & $48.4 \mathrm{abcd}$ & $56.17 \mathrm{abcd}$ & $2.8 \mathrm{a}$ & $2.9 \mathrm{a}$ & $3,1 \mathrm{a}$ & $91.7 \mathrm{a}$ & $100 \mathrm{a}$ & $100 \mathrm{a}$ \\
\hline
\end{tabular}

${ }^{\dagger}$ Para interacciones los promedios con letras semejantes son estadísticamente iguales, según la prueba de Tuckey al 95\%.

1//Valery.

2/ Barraganete. 
Cuadro 2. Promedio total de las tres cepas de Musaceas, registradas en la propagación vegetativa de Plátano y Banano con la aplicación de BAP y AIA

\begin{tabular}{llccc}
\hline & $\begin{array}{c}\text { Número } \\
\text { de brotes }\end{array}$ & $\begin{array}{c}\text { Longitud de } \\
\text { brotes }\end{array}$ & $\begin{array}{c}\text { Diámetro de } \\
\text { brotes }\end{array}$ & Supervivencia \\
\hline $\mathbf{C}_{\mathbf{0}}$ & $1.89 \mathrm{ab}^{\dagger}$ & $51.25 \mathrm{a}^{\dagger}$ & $3.09 \mathrm{a}^{\dagger}$ & $100 \mathrm{a}^{\dagger}$ \\
$\mathbf{C}_{\mathbf{1}}$ & $2.36 \mathrm{a}$ & $45.49 \mathrm{ab}$ & $2.67 \mathrm{ab}$ & $94.4 \mathrm{a}$ \\
$\mathbf{C}_{\mathbf{2}}$ & $2.00 \mathrm{ab}$ & $43.98 \mathrm{ab}$ & $2.72 \mathrm{ab}$ & $100 \mathrm{a}$ \\
$\mathbf{C}_{\mathbf{3}}$ & $1.84 \mathrm{ab}$ & $48.19 \mathrm{ab}$ & $3.03 \mathrm{a}$ & $97.2 \mathrm{a}$ \\
$\mathbf{C}_{\mathbf{4}}$ & $2.01 \mathrm{ab}$ & $43.76 \mathrm{ab}$ & $2.81 \mathrm{ab}$ & $94.4 \mathrm{a}$ \\
$\mathbf{C}_{\mathbf{5}}$ & $2.19 \mathrm{ab}$ & $47.48 \mathrm{ab}$ & $2.93 \mathrm{a}$ & $97.2 \mathrm{a}$ \\
$\mathbf{C}_{6}$ & $2.05 \mathrm{ab}$ & $41.20 \mathrm{~b}$ & $2.40 \mathrm{~b}$ & $94.4 \mathrm{a}$ \\
$\mathbf{C}_{7}$ & $1.81 \mathrm{~b}$ & $48.22 \mathrm{ab}$ & $2.87 \mathrm{ab}$ & $100 \mathrm{a}$ \\
$\mathbf{C}_{\mathbf{8}}$ & $1.97 \mathrm{ab}$ & $45.74 \mathrm{ab}$ & $2.89 \mathrm{a}$ & $97.2 \mathrm{a}$ \\
\hline
\end{tabular}

${ }_{\dagger}$ Los promedios con letras semejantes son estadísticamente iguales, según la prueba de Duncan al 95\%.

\section{DISCUSIÓN}

En las variables altura, diámetro y número de brotes se observó que la variedad banano Orito mostró los mayores promedios; superando a las otras variedades, (Conam, 2005) indica que en la población de una especie vegetal no existen individuos que tengan la misma información genética (ADN), lo que es conocido como variabilidad genética, lo que influye a la variación de las concentraciones hormonales en la regeneración de brotes, coincidiendo con Bidweell (1993), quien expresa que las hormonas provocan una gran variedad de efectos en las plantas, siendo uno de estos, estimular la emisión de brotes.

La concentración hormonal con mayor influencia en la emisión de brotes por cepa frente a todas las variedades fue la $\mathrm{C}_{1}$ con un promedio total de 2.36 brotes más que el testigo al que no se le aplicó estimulante hormonal. Con la combinación de 6-BAP y AIA los mejores resultados se obtuvieron con $\mathrm{C}_{5}$ y $\mathrm{C}_{6}$ con un promedio de 2.19 y 2.05 respectivamente, superando en brotes al testigo, demostrando que la combinación de estas dos hormonas influye significativamente en la emisión de brotes coincidiendo con lo indicado por Salisbury y Roos (2000) quienes mencionan que la relación auxina - citocinina es importante para controlar la dominancia apical, debido a que concentraciones altas favorecen el desarrollo de yemas y la concentraciones bajas favorecen la dominancia apical.

En la longitud de brotes la $\mathrm{C}_{0}$ alcanzó el mayor promedio, frente a todas las variedades mostrando un incremento de $51.25 \mathrm{~cm}$, esto se debe a la producción de un brote, favoreciendo a su desarrollo, debido a la alta disponibilidad de nutrientes.

Para el diámetro $\mathrm{C}_{0}$ presentó el mayor promedio en diámetro de brotes, con $3.09 \mathrm{~cm}$, las restantes concentraciones alcanzaron promedios entre 2.40 y $2.87 \mathrm{~cm}$, esto corresponde que a mayor presencia de número de brotes se distribuyen uniformemente los contenidos hormonales para favorecer a la longitud y al diámetro alcanzado por las mismas. Bidwell (1993), quien expresa que las hormonas provocan una gran variedad de efectos en las plantas; siendo una de estos el estimular la emisión de brotes.

Las variedades de plátano Barraganete y banano Orito mostraron el mayor porcentaje de supervivencia, con una diferencia del $8.33 \%$ más que el banano Valery, lo que puede deberse a los caracteres genéticos. Al respecto Sierra (1993) indica que cada una de las especies vegetales presenta características morfológicas que las hacen diferentes.

\section{CONCLUSIONES}

La concentración hormonal de $30 \mathrm{mg} \mathrm{L}^{-1} \mathrm{BAP}$ influyó positivamente en la emisión de brotes en cada variedad, seguida por la concentración $30 \mathrm{mg} \mathrm{L}^{-1} \mathrm{BAP}$ $+10 \mathrm{mg} \mathrm{L}^{-1}$ AIA con promedios de 2.36 y 2.19 respectivamente. Sin la aplicación de estos reguladores $\left(\mathrm{C}_{0}\right)$ se obtuvo el mayor promedio de altura de brotes en las tres variedades: banano var. Valery, var. Orito y plátano var. Barraganete.

\section{LITERATURA CITADA}

Conam 2005. Variabilidad Genética (en línea). Lima, PE. Consultado el 24 Oct. 2005. Disponible en: http://portalagrario.gov.pe/rrnn_gene.shtml.

Bidwell, R.G.S. 1993. Fisiología Vegetal. (Acción de las hormonas y Reguladores del crecimiento). Ontario, CA. A.G.T editor S. A. Primera edición. c.23. p. $598-619$.

Maluenda y Reyes, A. 2003. AUXINAS: química, biosíntesis de la auxina, fitohormonas, transporte, catabolismo auxínico (en línea). Valparaíso. CL. Consultado el 17 agos. 2005. Disponible en: http:// pdf.rincondelvago.com/auxinas.html

Méndez, F. 2003. Principios de propagación de las plantas (en línea). Universidad de la Molina. Lima, PE. Consultado el 22 abr. 2005. Disponible en: http:// www.lamolina.edu.pe/FACULTAD/agronomia/ horticultura/propagacion/fitohormonas/fmendez. doc.

Salisbury y Roos, C. 2000. Fisiología de las plantas. Paraninfo S. A. Thomson Editores. Madrid, ES. c. 17 . p. $581-585$; c. 18 . p. $610-616$; c. 22. p. $758-760$.

Sierra, L. E. 1993. El Cultivo de Banano. Producción y Comercio. Bogotá, CO. p. 121 - 125. 
Taiariol, D. R. 2000. Propagación Vegetativa (en línea). Consultado el 26 abr. 2004. Disponible en: http:// www.monografias.com/trabajos 13/propaveg/ propaveg.html

Vásquez, C., Orozco, A., Sánchez, E y Cervantes, V. 2000. La reproducción de las plantas: Semillas y Meristemos (en línea). Consultado el 28 mar. 2005. Disponible en: http://omega.ilce.edu.mx:3000/ sites/ciencia/volumen3/ciencia3/157/htm. 